\title{
Early Plasma Creatinine Values in Discordant Twins
}

\author{
Ilan Arad, Benjamin Bar-Oz, and Ofra Peleg \\ The Department of Neonatology, Hadassah University Hospital, Israel
}

\begin{abstract}
It has been suggested that impairment of placental perfusion prior to delivery may manifest in early postnatal increase of creatinine values. We hypothesized that the smaller of a discordant set of twins would have a higher initial plasma creatinine value and decided to measure early plasma creatinine levels in discordant twins in order to evaluate whether this value may serve as an index of impaired placental perfusion. Plasma creatinine, urea nitrogen and blood hematocrit values were simultaneously measured in 35 sets of twins during the first day of life. The sets of twins were divided into 2 groups according to birth weight difference. Thus, 18 sets of discordant twins with birth weight difference greater than $15 \%$ comprised the GT group and 17 sets of twins with birth weight difference less than or equal to $15 \%$ comprised the LE group. The differences between the values obtained within each group were analyzed using the Wilcoxon Signed Rank test. In the GT group the mean plasma creatinine level of the smaller twins was significantly higher than the level of the larger ones $(p=0.03)$, but there was no statistically significant difference between values obtained in twins of the LE group. The mean plasma urea level was higher in the larger twins of both groups, however only the difference in the GT group was statistically significant $(p=0.01)$. The mean hematocrit of the smaller twins was higher in both groups, but only the difference in the LE group was statistically significant ( $p=$ 0.02). Generally, there was a negative correlation between gestational age and early creatinine values. These results apparently support the notion that prenatal exposure to impaired placental perfusion may compromise the creatinine clearance of the fetus and result in higher early creatinine values. Since the creatinine values in our growth-retarded twins were within the normal range, no distinguishing line for evidence of a uterine-placental compromise could be drawn. Whether a certain early plasma creatinine value is suggestive or indicative of an intra-uterine hypoxic-ischemic insult, should be determined by documented instances of severe fetal compromise prior to delivery.
\end{abstract}

Fetal glomerular filtration rate increases with gestational age and is similar during intrauterine and extrauterine life (Arant, 1978; Coulthard, 1985; Wilkins, 1992). The evolvement of glomerular filtration rate is the result of different maturational processes including, among others, active nephrogenesis, changes in renal vascular resistance and the increasing function of the superficial nephrons (Guillery et al.,1998). Wladimiroff and Campbell (1974) have reported that in the abnormal pregnancy, for example, intrauterine growth retardation, there was a greater likelihood of a lower fetal urine production, and suggested that both a decreased renal size and an increased reabsorbtion of tubular fluid may account for the low urine flow.
Creatinine values are frequently used for the assessment of glomerular filtration rate in the neonate. However, since creatinine equilibrates across the placenta, the fetal or umbilical cord plasma values are believed to reflect maternal renal function and muscle mass rather than the renal function of the fetus or the newborn infant (Lao et al., 1989; Schwartz et al., 1984). As functional renal disturbances are often observed following perinatal asphyxia (Carter et al., 1993; Dauber et al., 1976; Perlman et al., 1989), it has been suggested that impairment of placental perfusion occurring prior to delivery may manifest in an early postnatal (first day) increase of creatinine values (Altshuler, 1997). Impaired placental perfusion is often the cause for the intrauterine growth retardation of a discordant twin and is frequently associated with sub-optimal fetal oxygenation and decreased renal mass (Bernischke \& Gille, 1977; Brayn, 1999). We accordingly hypothesized that the smaller of a discordant set of twins would have a higher initial blood creatinine value. We therefore decided to measure early plasma creatinine levels in discordant twins and to evaluate whether this value may serve as an index of prenatal exposure to hypoxic-ischemic insult.

\section{Patients and Methods}

Thirty-five sets of twins delivered at the Hadassah University Hospital in Jerusalem between October, 1999 and April, 2000 were included in the study. None of them suffered from asphyxia or required intensive care, and there were none with clinical signs of twin to twin transfusion. Venous blood was analyzed for plasma creatinine, urea nitrogen and hematocrit values from both twins during the first day of life $(7.5-26$ hours) as part of the routine work-up. Obstetric and perinatal data were obtained from hospital files. Creatinine and urea nitrogen were determined by the dry-slide technology on a Kodak Etachem Analyzer. Gestation was assessed by maternal menstrual history. The twins were either breast fed or bottle fed $70-80 \mathrm{~mL} / \mathrm{kg}$ during the first day of life. The twins were divided into 2 groups according to the difference between the birth weights of each set. There were 18 sets of discordant twins with birth weight difference greater than 15\% (the GT

Address for correspondence: Benjamin Bar-Oz, M.D., Department of Neonatology., Hadassah University Hospital, Mt. Scopus, 91240, Jerusalem,Israel.Email: baroz@hadassah.org.il 
group) and 17 sets of twins with birth weight difference of less or equal to $15 \%$ (the LE group).

\section{Statistical Analysis}

The levels and distributions of the continuous variables of plasma creatinine, urea nitrogen and blood hematocrit were measured in all the twins to determine the appropriateness of tests based on the normal approximation. For both creatinine and urea nitrogen the distribution showed a skewness that rendered the use of $t$ tests in the samples of 17 of the twins questionable. We therefore decided to apply the non-parametric Wilcoxon Signed Rank test. The rationale for any difference in creatinine levels calls for a one-sided hypothesis. Accordingly, the $p$-values reported for this variable are one-sided. The $p$-values reported for all other tests of significance are 2 -sided. The association between creatinine and gestational age was analyzed using linear regression. Time lapse between the first and second born twin had a highly skewed distribution. Comparison between the groups was made by means of the Mann Whitney test.

\section{Results}

Obstetric and perinatal variables are summarized in Table 1. By definition the difference between twins' birth weights was larger among the GT group. As might be expected the differences between twins in the GT group also applied to the mean length and head circumference.

The time lapse between the delivery of twin A and B in the LE group was $6.2 \pm 9.3$ minutes and $4.1 \pm 4.9$ in the GT group $(p=0.85)$. There was also no difference between the groups with regard to placental chorionicity. In each group, 13 pairs were dichorionic, one pair was monochorionic and there were no available data of the remaining pairs.

The biochemical and hematological results are summarized in Table 2. In the GT group the mean plasma creatinine level of the smaller twin of each set was significantly higher than the level of the larger one $(p=0.03)$, but there was no statistically significant difference between creatinine levels in twins of the LE group. Plotting the growth discordance versus the creatinine discordance of the total sample of infants revealed a positive correlation which was just short of statistical significance $(R=.31 ; p=0.07)$, (Figure 1a) however no significant correlation was demonstrated within the particular study groups (Figure 1b,c). In the LE group the creatinine level of the first born twin was $84.1 \pm 11.4 \mu \mathrm{mole} / \mathrm{L}$ and $80.1 \pm 8.1 \mu$ mole/ $\mathrm{L}$ in the

\section{Table 1}

Obstetric and Perinatal Variables.*

\begin{tabular}{lcccc}
\hline & \multicolumn{2}{c}{$\begin{array}{c}\text { GT group } \\
\text { ( } n=18 \text { pairs })\end{array}$} & \multicolumn{2}{c}{$\begin{array}{c}\text { LE group } \\
(n=17 \text { pairs })\end{array}$} \\
& $\begin{array}{l}\text { Large } \\
\text { twin }\end{array}$ & $\begin{array}{c}\text { Small } \\
\text { twin }\end{array}$ & $\begin{array}{c}\text { Large } \\
\text { twin }\end{array}$ & $\begin{array}{c}\text { Small } \\
\text { twin }\end{array}$ \\
\hline Birth weight (Kg) & $2.48 \pm 0.46$ & $1.88 \pm 0.43$ & $2.51 \pm 0.42$ & $2.31 \pm 0.41$ \\
Wt. Difference (\%) & \multicolumn{2}{c}{$24.7 \pm 7.9$} & \multicolumn{2}{c}{$7.9 \pm 4.3$} \\
Gestational age (weeks) & $36.3 \pm 2.2$ & \multicolumn{2}{c}{$36.4 \pm 2.2$} \\
C-section & 12 & 12 & 9 & 9 \\
Female & 7 & 12 & 4 & 6 \\
Apgar 1' & $8.7 \pm 0.8$ & $8.6 \pm 1.0$ & $8.8 \pm 0.7$ & $8.6 \pm 0.6$ \\
Apgar 5' & $9.7 \pm 0.5$ & $9.7 \pm 0.6$ & $9.9 \pm 0.2$ & $9.8 \pm 0.4$ \\
Length (cm) & $45.8 \pm 2.6$ & $43.1 \pm 3.0$ & $47.1 \pm 1.4$ & $45.9 \pm 1.8$ \\
Head circ. (cm) & $32.6 \pm 1.6$ & $30.6 \pm 1.8$ & $32.8 \pm 1.3$ & $32.2 \pm 1.6$ \\
Sampling time (hours) & \multicolumn{2}{c}{$14.8 \pm 5.1$} & \multicolumn{2}{c}{$15.4 \pm 3.4$} \\
\hline
\end{tabular}

* Values are mean \pm standard deviation.

second. In the GT group the creatinine level of the first born twin was $87.1 \pm 19.9 \mu$ mole/ $\mathrm{L}$ and $86.4 \pm 18.9$ $\mu \mathrm{mole} / \mathrm{L}$ in the second. In both groups the difference was not statistically significant $(p=0.164$ and 0.433 , respectively). When taken together there was no significant difference between the creatinine levels of the first and second born twins $(p=0.14)$.

The mean plasma urea level was higher in the larger twins in both the GT and the LE groups, however only the difference in the GT group was statistically significant $(p=$ 0.01 ). The mean hematocrit of the smaller twins was higher in both the GT and the LE groups. The difference in the LE group was statistically significant $(p=0.02)$. There was a negative correlation between gestational age and the early plasma creatinine levels of the combined study groups. In the larger twins of both groups combined, the correlation was statistically significant $(R=-.393 ; p=0.02)$, but this was not the case with the smaller twins $(R=-.238 ; p=0.17)$.

In analysis of the GT group, there was a statistically significant negative correlation between early creatinine levels and gestational age, both for the smaller and the larger twins $(R=-.592 ; p=0.01, R=-.574 ; p=0.013$, respectively). In the LE group there was a insignificant negative correlation between creatinine levels and gestational age of the larger twins $(R=-.140 ; p=0.59)$, however the correlation of the smaller twins was positive and just short of statistical significance $(R=.455 ; p=0.067)$.

\section{Table 2}

Plasma Creatinine, Urea Nitrogen and Blood Hematocrit Values.*

\begin{tabular}{|c|c|c|c|c|c|c|}
\hline & \multicolumn{3}{|c|}{ GT group (18 Pairs) } & \multicolumn{3}{|c|}{ LE group (17 pairs) } \\
\hline & Large twin & Small twin & $P$ value & Large twin & Small twin & $P$ value \\
\hline Creatinine ( $\mu \mathrm{mol} / \mathrm{L})$ & $84.1 \pm 19.7$ & $89.4 \pm 18.8$ & 0.03 & $83.5 \pm 11.4$ & $80.7 \pm 8.3$ & 0.33 \\
\hline Urea (mmol/L) & $3.8 \pm 1.5$ & $3.1 \pm 1.0$ & 0.01 & $3.5 \pm 1.3$ & $3.1 \pm 0.7$ & 0.52 \\
\hline Hematocrit (\%) & $56.8 \pm 8.5$ & $58.8 \pm 5.8$ & 0.35 & $55.6 \pm 6.4$ & $59.7 \pm 7.6$ & 0.02 \\
\hline
\end{tabular}

* Values are mean \pm standard deviation. 


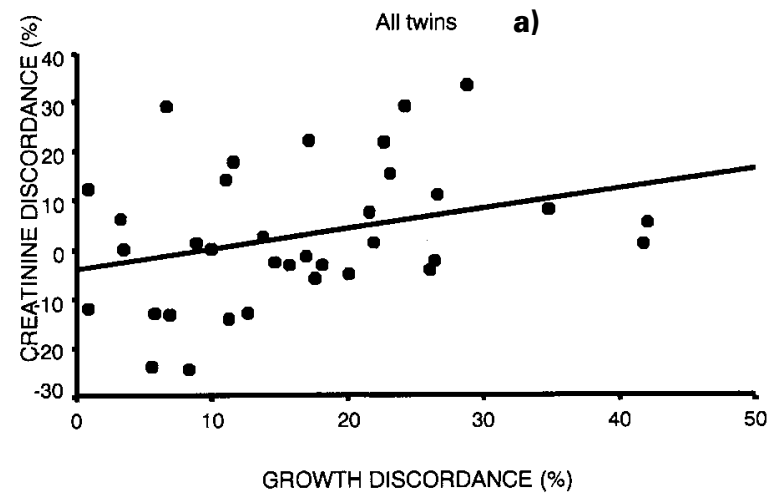

For creatinine: Smaller - larger. Percentages are of larger twin values

Regression slope $=0.41, p=0.07, r=0.31$

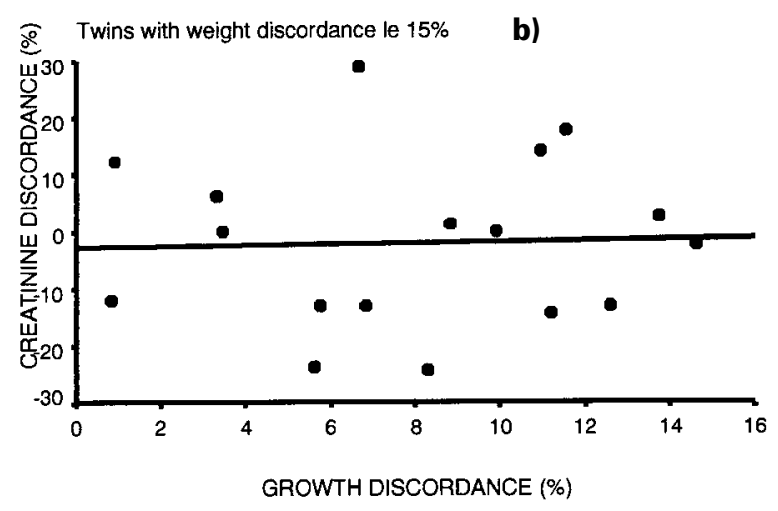

For creatinine: Smaller - larger. Percentages are of larger twin values

Regression slope $=0.08, p=0.93, r=0.02$

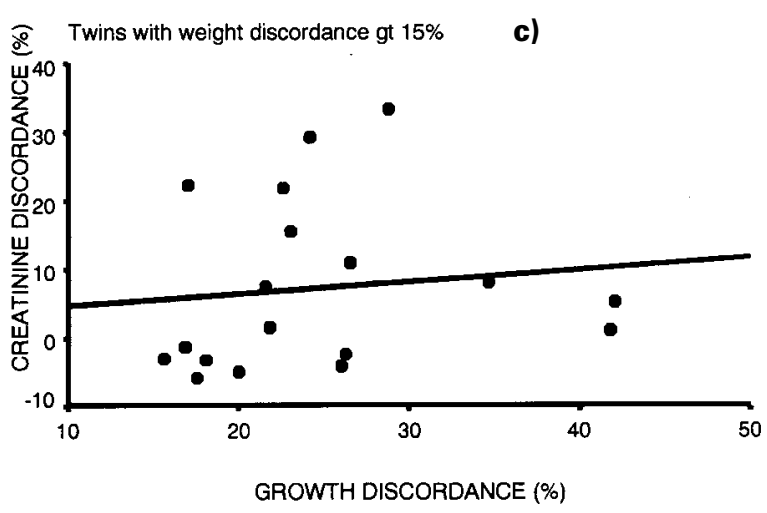

For creatinine: Smaller - larger. Percentages are of larger twin values

Regression slope $=0.17, p=0.66, r=0.11$

\section{Figure 1}

Creatinine discordance vs. growth discordance in a) Combined groups (GT + LE), b) LE group, c) GT group.

\section{$\overline{\text { Discussion }}$}

Statistically significant higher initial creatinine values were observed in the smaller of the discordant (GT group) twins compared with their larger sibling. No such difference was found in the LE group where the weight difference between the twins in each set was $15 \%$ or less. The correlation between growth discordance and plasma creatinine discordance of the total sample of infants was just short of statistical significance. The lack of correlation within the GT group may have been the result of too small a number of infants studied.

Assuming that interference of uterine-placental perfusion accounted for the fetal growth retardation in at least, some sets of the discordant twins, these results apparently support the notion that prenatal exposure to hypoxic-ischemic conditions may compromise the creatinine clearance of the fetus, and result in higher early creatinine values (Altshuler, 1997). The higher creatinine values in our growth-retarded twins, were within the normal range (Rudd et al., 1983) and no distinguishing line for evidence of a uterine-placental compromise could be drawn. This finding is not unexpected since the growth retarded twins had good Apgar scores and did not seem to suffer from stressful conditions of high severity prior to delivery. In some cases, a perfusion problem might have not been the reason for the weight difference. It could be caused by other factors (e.g., genetic). An alternative explanation for our observation may be the presence of a decreased renal mass in the growth-retarded twins with consequent lower renal clearance ability.

Placental chorionicity has been implicated in the discordant growth of twins (Charlemaine et al., 2000; Hatkar \& Bhide, 1999 ). While growth discordance in both monochorionic and dichorionic pairs may occur due to unequal sharing of the placental parechyma secondary to peripheral insertion of the cord of the smaller twin, hematological and biochemical differences may be minimized in monochorionic pairs by the intermixing of fetal blood through inter-fetal vascular connections. Since both of our study groups had a high and similar prevelance of dichorionic pairs, chorionicity does not appear to have a major impact on our results.

Our data are in accordance with previous results indicating that initial creatinine levels are inversely related to gestational age (Bueva \& Guignard, 1994; Guignard \& Drukker, 1999). These higher levels were previously attributed to poor clearance of a large creatinine load inherited from the mother (Bueva \& Guignard, 1994). Miall et al. (1999) have recently challenged this assumption and suggested that plasma creatinine values at the time of birth merely reflect normal maternal levels. However, maternal levels were not given in their study and the initial creatinine values were almost double their cited maternal references. Guignard and Drukker (1999) have demonstrated an ongoing fetal-maternal creatinine equilibration during the second half of gestation. Addressing the riddle of the high plasma creatinine levels in term and especially in preterm infants, they suggest that once the umbilical cord is severed, the fetal-maternal balance is disturbed with a transient occurrence of tubular creatinine reabsorption across leaky immature tubular and vascular structures. In view of the high creatinine values measured in cord blood (Henderson et al., 1998), we suggest that such a phenomenon probably occurs already in-utero at the commencement of labor, and may be accentuated in pathological conditions (Altshuler, 1997).

In contrast to the creatinine levels, the blood urea nitrogen levels of the larger discordant twins in our study were 
significantly higher than levels of the smaller ones while there was no significant difference between twins of the LE group. Urea levels may be affected by several factors. In our case, the higher values probably reflect the more effluent uterine-placental flow of nutrients, including protein, to the larger twin.

Lower ferritin and higher transfferin levels were previously demonstrated in instances of intrauterine hypoxia, that is, maternal toxemia or diabetes, and were attributed to enhanced hematopoiesis occurring in these conditions (Chokalingam et al., 1987; Georgieff et al., 1992). As expected, higher hematocrit values were found in the smaller twins of both study groups, but unexpectedly, the difference was statistically significant only in the LE group. We do not have an apparent explanation for this finding, however, the initial hematocrit values in the newborn could be also affected by factors such as lower placement of the newborn prior to cord clamping or delayed clamping of the cord. These were not accounted for in our study.

\section{Conclusion}

Higher early plasma creatinine levels were demonstrated in smaller discordant twins when compared with levels of their larger siblings. This finding may be due to an impaired uterine-placental perfusion of the growth-retarded twins or a consequence of a decreased renal mass. Whether a certain early plasma creatinine value is suggestive or indicative of an intra-uterine hypoxic-ischemic insult, should be determined in documented instances of severe fetal compromise prior to delivery.

\section{$\overline{\text { References }}$}

Altshuler, G. (1997). The relationship of placental pathology to causation of detrimental pregnancy outcome. In D. K. Stevenson \& P. Sunshine (Eds.), Fetal \& neonatal brain injury ( $2^{\text {nd }}$ ed.; pp. 585-601). Oxford: Oxford University Press.

Arant, B. S., Jr. (1978). Developmental patterns of renal function maturation compared in the human neonate. The Journal of Pediatrics, 92, 705-712.

Bernischke, K., \& Gille, J. (1977). Placental pathology and asphyxia. In L. Gluck (Ed.), Intrauterine asphyxia and the developing fetal brain (p 117). Chicago, IL.: Year Book Medical Publishers Inc.

Bryan, E. M. (1999). Twins. In J. M. Renie \& N. R. C. Roberton (Eds.), Textbook of neonatology (3rd ed., pp. 409-416). Sidcup, Kent: Churchill Livingstone.

Bueva, A., \& Guignard, J. P. (1994). Renal function in preterm neonates. Pediatric Research, 36, 572-577.

Carter, B. S., Haverkamp, A. D., \& Merenstein, G. B. (1993). The definition of acute perinatal asphyxia. Clinics in Perinatology, 20(2), 287-303.

Charlemaine, C., Duyme, M., Ville, Y., Aurengo, A., Tremblay, R., Frydman, R., \& Pons, J. C. (2000). Fetal biometric parameters, twin type and birth weight difference. A longitudinal study. European Journal of Obstetrics, Gynecology, and Reproductive Biology, 93, 27-32.
Chockalingam, U. M., Murphy, E., Ophoven, J. C., Weisdorf, S. A., \& Georgieff, M. K. (1987). Cord transferrin and ferritin values in newborn infants at risk for prenatal uteroplacental insufficiency and chronic hypoxia. The Journal of Pediatrics, 111, 283-286.

Coulthard, M. G. (1985). Maturation of glomerular filtration in preterm and mature babies. Early Human Development, 11, 281-292.

Dauber, I. M., Krauss, A. N., Symchych, P. S., \& Auld, P. A. (1976). Renal failure following perinatal anoxia. The Journal of Pediatrics, 88, 851-855.

Georgieff, M. K., Schmidt, R. L., Mills, M. M., Radmar, W. J., \& Widness, J. A. (1992). Fetal iron and cytochrome c status after intrauterine hypoxemia and erythropoietin administration. American Journal of Physiology, 262, R485-R491.

Guignard, J. P., \& Drukker, A. (1999). Why do newborn infants have a high plasma creatinine? Pediatrics, [Online-serial], 103(4), Available URL: http://pediatrics.org/cgi/content/full/ 103/4/e49.

Guillery, E. N., Nuyt, A. M., \& Robillard, J. E. (1998). Functional development of the kidney in utero. In R. A. Polin $\&$ W. W. Fox (Eds.), Fetal and Neonatal Physiology (2 ${ }^{\text {nd }}$ ed.; pp. 1560-1573). St. Louis, MO: WB Saunders.

Hatkar, P. A., \& Bhide, A.G. (1999). Perinatal outcome of twins in relation to chorionicity. Journal of Postgraduate Medicine, 45, 33-37.

Henderson, M. J., Brownlee, K. G., Turner, A. J., \& Brocklebank, J. T. (1998). Serial changes in plasma creatinine in the first week of life of premature babies. Pediatric Nephrology, 12, C90.

Lao, T. T., Loong, E. P., Chin, R. K., \& Lam, Y. M. (1989). Renal function in the newborn: Newborn creatinine related to birth weight, maturity and maternal creatinine. Gynecologic and Obstetric Investigation, 28, 70-72.

Miall, L. S., Henderson, M. J., Turner, A. J., Brownlee, K. G., Brocklebank, J. T., Newell, S. J., \& Allgar, V. L. (1999). Plasma creatinine rises dramatically in the first 48 hours of life in preterm infants. Pediatrics, [On-line serial], 104(6), Available URL: http://intl.pediatrics.org/cgi/content/full/ 104/6/e76.

Perlman, J. M., Tack, E. D., Martin, T., Shakelford, G., \& Amon, E. (1089). Acute systemic organ injury in term infants after asphyxia. American Journal of Diseases of Children, 143, 617-620.

Rudd, P. T., Hughes, E. A., Placzek, M. M., \& Hodes, D. T. (1983). Reference ranges for plasma creatinine during the first month of life. Archives of Disease in Childhood, 58, 212-215.

Schwartz, G. J., Feld, L. G., \& Langford, D. J. (1984). A simple estimate of glomerular filtration rate in full term infants during the first year of life. The Journal of Pediatrics, 104, 849-854.

Wilkins, B. H. (1992). Renal function in sick very low birthweight: I. Glomerular filtration rate. Archives of Disease in Childhood, 67, 1140-1145.

Wladimiroff, J. W., \& Campbell, S. (1974). Fetal urine-production rates in normal and complicated pregnancy. Lancet, 1, 151-154. 